\title{
PERDAS DE CARBONO E NITROGÊ NIO COM APLICAÇÃO DE DEJETO LÍQUIDO BOVINO EM LATOSSOLO MUITO ARGILOSO SOB PLANTIO DIRETO E CHUVA NATURAL ${ }^{(1)}$
}

\author{
Adriana Timofiecsyk ${ }^{(2)}$, Nerilde Favaretto ${ }^{(3)}$, Volnei Pauletti ${ }^{(3)}$ \& J eferson Dieckow ${ }^{(3)}$
}

\begin{abstract}
RESUMO
O transporte de poluentes via escoamento superficial em áreas agrícolas, principalmente na forma solúvel, é considerado um problema ambiental, mesmo em sistemas conservacionistas de preparo do solo. $O$ objetivo deste trabalho foi avaliar as perdas de carbono orgânico e nitrogênio no escoamento superficial,

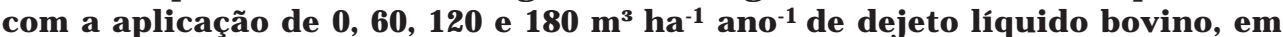
Latossolo muito argiloso, com declividade de $10 \%$, sob plantio direto e chuva natural, na região de Castro - PR. A aplicação do dejeto líqui do bovino não alterou o carbono orgânico total, porém diminuiu as perdas de amônio e nitrato até a dose de $120 \mathrm{~m}^{3} \mathrm{ha}^{-1}$ ano-1, o que indica ser esta a dose máxi ma recomendada para solo muito argi loso, declive moderadamente ondulado, baixas precipitações e com no mínimo uma semana de intervalo entre a aplicação do dejeto e a ocorrência de chuvas. As concentrações médias ponderadas de amônio e nitrato no escoamento superficial aumentaram com a aplicação de dejeto. As concentrações de amônio estiveram muito acima do máxi mo permitido pela legi slação brasileira, inclusive sem aplicação de dejeto, o que indica a necessidade de práticas que evitem a entrada do escoamento em corpos de água, mesmo em sistemas conservacionistas.
\end{abstract}

Termos de indexação: escoamento superficial, qualidade da água, nutrientes, nitrato, amônio, adubação orgânica.

(1) Parte da Dissertação de Mestrado do primeiro autor apresentada ao Programa de Pós Graduação em Ciência do Solo (PPGCS), da Universidade Federal do Paraná - UFPR. Recebido para publicação em 30 de novembro de 2011 e aprovado em 02 de outubro de 2012.

(2) Eng.-Agrônoma, Mestre em Ciência do Solo. PPGCS, UFPR. Rua dos Funcionários, 1540. CEP $80035-050$ Curitiba (PR). Email: agro_adriana@yahoo.com.br

(3) Professor Adjunto do Departamento de Solos e Engenharia Agrícola, UFPR. E-mails: nfavaretto@ufpr.br; jefersondieckow@ufpr.br; vpauletti@ufpr.br 


\author{
SUMMARY: CARBON AND NITROGEN LOSSES WITH LIQUID CATLLE \\ MANURE FROM A CLAYEY OXISSOL UNDER NO-TILL AND \\ NATURAL RAINFALL
}

\begin{abstract}
Thetransport of poll utants via runoff in agricultural areas, mainly in solubleforms, is an environmental problem, even in conservation tillagesystems. Theobjectiveof this study was to evaluatel osses of nitrogen and organic carbon in runoff, after applications of $0,60,120$, and $180 \mathrm{~m}^{3} \mathrm{ha}^{-1} \mathrm{yr}^{-1}$ of cattlesl urry toa dayey Oxisol, with $10 \%$ slopeunder no-tillageand natural rainfall, in theregion of Castro- PR. Theapplication of cattleslurry did not affect total organic carbon in runoff but ammonium and nitratelosses werereduced by thefertilization with 120 $\mathrm{m}^{3} \mathrm{ha}^{-1} \mathrm{yr}^{-1}$ of slurry, indicating this as themaximum doserecommended for soils with a high clay content, moderatesl opeand low rainfall, with at least oneweek interval between manure application and rainfall. The weighted average concentrations of ammonium and nitrate increased with manureapplication. Theammoni um concentrations exceeded thethreshol d established by the Brazilian law by far, even in the treatment without slurry application, indicating the need for practices that prevent runoff from entering watercourses, even in conservation tillagesystems.
\end{abstract}

Index terms: runoff, water quality, nutrient, nitrate, ammonium, organic fertilizer.

\section{INTRODUÇÃO}

A produção animal no Brasil cresce a cada ano, o que, consequentemente, gera uma grandequantidade de dejetos, os quais vêm sendo utilizados na agricultura. No entanto, o uso inadequado desse resíduo pode causar problemas ambientais, entre os quais a contaminaçãodas águas por diversos poluentes (Sharpley, 1995; Hooda et al., 2000; Shigaki et al., 2006; Kay et al., 2009).

O nitrogênio $(N)$ - nutriente essencial para o crescimento de plantas e, portanto, intensi vamente aplicado no sol o em sistemas de produção agrícola promove também o crescimento de organismos em ecossistemas aquáticos, al terando a qual idade da água (Correll, 1998; Daniel et al., 1998; Smith et al., 1999). Além da eutrofização, o N pode causar problemas à saúde humana, como, por exemplo, a síndrome do bebêazul ou meta-hemogl obinemia, a qual é causada pel o $\mathrm{N}$ na forma de $\mathrm{NO}_{3}{ }^{-}$(Smith et al., 1990; Dinnes et al., 2002). O nitrogênio amoniacal (amônia + amônio) também é prejudicial tanto para a saúde humana como para a vida aquática (Smith et al., 1990). Por essas razões, as concentrações de $\mathrm{N}$ nas formas de $\mathrm{NO}_{3}{ }^{-}$e $\mathrm{NH}_{4}{ }^{+}$são controladas por legislação, a fim de assegurar a qualidade das águas.

O carbono $(C)$ também é um el emento poluente; sua presença nos corpos de água altera a disponi bilidade de oxigênio dissolvido em função da decomposição da matéria orgânica pelos microrganismos aquáticos. Além disso, a presença de C solúvel em reservatórios é de grande preocupação em razão dos tri-hal ometanos, compostos cancerígenos formados no processo de cloração, em sistemas de tratamento deágua para abastecimento humano (Kay et al., 2009).
Em áreas agrícolas, a qualidade da água é grandemente afetada pelo enriquecimento dos corpos de água com nutrientes via escoamento superficial. O sistema de plantio direto (SPD), por manter a superfície do solo coberta, é uma importante ferramenta para control ar os processos de perda de soloe, consequentemente, dos poluentes associados aos sedimentos, porém nem sempre é uma ferramenta eficaz no controle de perda deágua (Cogo et al., 2003). A menor eficácia no controle dessa perda deve-se ao fato de a capacidade de infiltração do sol o ser finita, independentemente do sistema de preparo. Portanto, mesmo em sistemas conservacionistas, onde ocorre redução de perda de solo (Derpsch et al., 1991), deve-se considerar o potencial de transporte de poluentes via escoamento superficial (Resck et al., 1980; Silva et al., 2005), princi pal mente de poluentes na forma solúvel (Sharpley et al., 1994; Bertol et al., 2005). Assim, o plantio direto (PD) deve estar aliado a outras práticas conservacionistas, como terraceamento, plantio em nível, rotação de culturas, entre outros, a fim de efetivamente se tornar um sistema conservacionista de sol o e água (Bertol et al., 2012).

O uso de dejetos na agricultura tem grande potencial de contaminação das águas (Bertol et al., 2007a; Mori et al., 2009), pois eles são ricos em nutrientes e matéria orgânica, o que aumenta consi deravel mente os seus níveis no solo, el evando o potencial de perdas pelo escoamentosuperficial (Pote et al., 1999).

O objetivo deste trabalho foi avaliar o efeito da aplicação de dejetolíquido bovino em Latossolo muito argil loso sob plantio direto echuva natural, na região dos Campos Gerais do Paraná, sobre as perdas de carbono orgânico total e de nitrogênio no escoamento superficial. 


\section{MATERIAL E MÉTODOS}

O presente trabal ho foi desenvolvido em Castro $\mathrm{PR}$, na unidade experimental da Fundação $A B C$, numa área com decl ividade de 9,6\%, com solo classificado como Latossolo Bruno distrófico típico (E mbrapa/ Fundação $A B C, 2001$ ), textura muito argilosa, sob plantio direto (PD) há mais de 15 anos. O clima da região é classificado como $\mathrm{Cfb}$, segundo $\mathrm{K}$ öppen, com preci pitação pluvial média anual de $1.554 \mathrm{~mm}$, série histórica de 1954 a 2001 (IAPAR, 2012). Os dados de precipitação pluvial mensal coletados na estação meteorológica da F undação $A B C$, bem como a média histórica (IAPAR, 2012), encontram-seno quadro 1.

Os atributos quími cos efísicos foram determinados antes da instalação do experimento em quatro profundidades, sendoas médias ponderadas, na camada de 0-20 cm: $\mathrm{pH}$ em $\mathrm{CaCl}_{2}=5,4 ; \mathrm{Al}^{3+}=0 \mathrm{cmol}_{\mathrm{c}} \mathrm{dm}^{-3}$; $\mathrm{H}+\mathrm{Al}=4,6 \mathrm{cmol}_{\mathrm{c}} \mathrm{dm}^{-3} ; \mathrm{Ca}^{2+}=5,1 \mathrm{cmol}_{\mathrm{c}} \mathrm{dm}^{-3}$; $\mathrm{Mg}^{2+}=1,4 \mathrm{cmol}_{\mathrm{c}} \mathrm{dm}^{-3} ; \mathrm{K}^{+}=0,23 \mathrm{cmol}_{\mathrm{c}} \mathrm{dm}^{-3} ; \mathrm{P}$ (Mehlich-1) $=4 \mathrm{mg} \mathrm{dm}^{-3} ; \mathrm{C}=25,7 \mathrm{~g} \mathrm{dm}^{-3} ; \mathrm{CTC}_{\mathrm{pH} 7}=$ $11,3 \mathrm{cmol}_{\mathrm{c}} \mathrm{dm}^{-3} ; 60 \%$ de saturação por bases; $701,4 \mathrm{~g}$ $\mathrm{kg}^{-1}$ de argila; $115,5 \mathrm{~g} \mathrm{~kg}^{-1}$ de silte; $187,02 \mathrm{~g} \mathrm{~kg}^{-1}$ de areia; diâmetro médio ponderado de agregados = $2,92 \mathrm{~mm}$; densidade de solo $=1,04 \mathrm{~g} \mathrm{~cm}^{-3}$; microporosidade $=42,62 \%$; macroporosidade $=16,53 \%$; econdutividadehidráulica saturada $=5,23 \mathrm{~mm} \mathrm{~h}^{-1}$.

Os tratamentos, distribuídos no delineamento em blocos casualizados (quatro blocos), consistiram de uma testemunha (sem aplicação de dejeto) etrês doses de dejeto: $60,120 \mathrm{e}^{180} \mathrm{~m}^{3} \mathrm{ha}^{-1} \mathrm{ano}^{-1}$, sendo estas doses aplicadas metade no plantio das culturas deinvernoe metade nas de verão, em superfície, entre as linhas de plantio e sem incorporação, com regadores

Quadro 1. Precipitação pluviométrica mensal
ocorrida em Castro- Paraná no período de maio/
2006 a abril/2008 e médias históricas de 1954 a
2001

\begin{tabular}{|c|c|c|c|c|}
\hline Mês ${ }^{(1)}$ & 2006 & 2077 & 2008 & História \\
\hline & & $-\mathrm{m}$ & 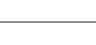 & \\
\hline J aneiro & nd & 192,5 & 103,6 & 186,5 \\
\hline Fevereiro & nd & 168 & 90 & 161,0 \\
\hline Março & nd & 86 & 123,8 & 137,8 \\
\hline Abril & nd & 61,8 & 200,2 & 101,3 \\
\hline Maio & 8,3 & 87,8 & nd & 116,3 \\
\hline J unho & 23,5 & 6,5 & nd & 117,7 \\
\hline J ulho & 18,5 & 134 & nd & 95,8 \\
\hline Agosto & 40,5 & 18,6 & nd & 78,9 \\
\hline Setembro & 132,5 & 31,8 & nd & 135,5 \\
\hline Outubro & 93,3 & 54 & nd & 152,7 \\
\hline Novembro & 115,5 & 119 & nd & 119,2 \\
\hline Dezembro & 91,6 & 277,8 & nd & 151,0 \\
\hline Total & 524,0 & 1237,0 & 518,0 & 1554,0 \\
\hline
\end{tabular}

(1) IAPAR (2012); nd=não determinado manuais, num sistema de rotaçãoaveia-preta, milho, aveia-preta e soja. O dejeto aplicado (Quadro 2) foi proveniente depropriedade produtora degado leiteiro, de sistema confinado, sendo a al imentação baseada em silagem, sal mineral eração. Foram adicionados via dejeto, para a dose de $60 \mathrm{~m}^{3} \mathrm{ha}^{-1} \mathrm{ano}^{-1}$, nos dois anos deexperimento, $196 \mathrm{~kg} \mathrm{ha}^{-1}$ de $\mathrm{N}$ total, $76 \mathrm{~kg} \mathrm{ha}^{-1}$ deP total e $304 \mathrm{~kg} \mathrm{ha}^{-1}$ de K total. Além da adubação orgânica, aplicou-se adubo mineral (187, 66 e $98 \mathrm{~kg}$ ha $^{-1}$ de N, P e K, respectivamente, nos dois anos de experimento), simulando a prática dos agricultores da região, os quais, de modo geral, aplicam adubo mineral independentemente da dose de dejeto. As culturas adubadas foram mil ho na safra 2006/2007 e soja na safra 2007/2008. Para a cultura do mil ho foram aplicados 187, 49 e $67 \mathrm{~kg} \mathrm{ha}^{-1}$ de N, P e K, respectivamente, via formulados 14-28-00 na semeadura e 25-00-25 em cobertura. Para a cultura da soja, aplicaram-se 0, 17 e $31 \mathrm{~kg} \mathrm{ha}^{-1}$ de N, P eK, respectivamente, via formulado 00-20-20.

As parcelas $(3,5 \mathrm{~m}$ de largura $\times 9 \mathrm{~m}$ de comprimento) foram instaladas em maio de 2006, com área de $29,75 \mathrm{~m}^{2}$, delimitadas por chapas galvanizadas de 0,10 m dealtura, introduzidas a 0,05 $\mathrm{m}$ no solo. Na parte inferior da parcela, as chapas foram dispostas em "V", a fim de canalizar o escoamento superficial para um cano de PVC de 100 $\mathrm{mm}$, que a conduzia para um reservatório com capacidade de $0,06 \mathrm{~m}^{3}$.

No período de 11/05/2006 (instalação do experimento) até 30/04/2008 foram realizadas 37 coletas, as quais foram feitas após cada chuva que produzisse escoamento. A primeira chuva que gerou escoamento, após a instal ação do experimento, foi em setembro/2006. O intervalo entrea aplicação do dejeto ea primeira chuva com formação de escoamento foi de130, 11, 5 e 10 dias, respectivamente para as safras deinverno2006, verão2006/2007, inverno 2007 everão 2007/2008.

Quanto às análises de nutrientes solúveis,

\section{Quadro 2. Matéria seca (MS) e teores totais de nitrogênio $(N)$, fósforo $(P)$ e potássio $(K)$ dos dejetos utilizados nas quatro aplicações ocorridas no período de maio de 2006 a maio de 2008}

\begin{tabular}{|c|c|c|c|c|}
\hline Data de aplicação & MS $^{(\mathbf{1})}$ & $N^{(2)}$ & $\mathbf{P}^{(2)}$ & $K^{(2)}$ \\
\hline & & - & & \\
\hline $11 / 05 / 06$ & 90,5 & 1,82 & 0,77 & 3,46 \\
\hline 05/10/06 & 75,5 & 1,74 & 0,61 & 2,37 \\
\hline 04/05/07 & 43,3 & 0,95 & 0,41 & 1,48 \\
\hline $30 / 11 / 07$ & 89,6 & 2,00 & 0,73 & 2,80 \\
\hline
\end{tabular}

(1)Teor de matéria seca determinado por gravimetria; (2)Teor em base úmida calculado pelo teor determinado em base seca. $\mathrm{O}$ teor de N foi determinado pelo método Dumas por combustão; o teor de $\mathrm{P}$, por digestão com ácido clorídrico e colorimetria; e o K, por digestão com ácido nitro-clorídrico e espectrofotometria de absorção atômica (Martins \& Reissmann, 2007). 
procedeu-seà filtragem das amostras em um filtro de membrana deéster decel ulosecom $0,45 \mu \mathrm{m}$. O nitrato $\left(\mathrm{NO}_{3}{ }^{-}\right)$e amônio $\left(\mathrm{NH}_{4}{ }^{+}\right)$foram determinados por espectrometria pelo método do ácido cl orídrico e pelo método do fenato, respectivamente, sob comprimento de onda de 220 e $275 \mathrm{~nm}$ para o $\mathrm{NO}_{3}$ - e $640 \mathrm{~nm}$ para o $\mathrm{NH}_{4}{ }^{+}$(APHA, 1995). O teor de carbono orgânico total (COT) foi determinado nas amostras nãofiltradas, de acordo com o método de refluxo aberto para determinação da demanda química de oxigênio, modificado por Boyd \& Tucker (1992).

A concentração média ponderada foi obtida multiplicando-se a concentração pelo volumedeágua perdido em cada coleta; esses produtos foram somados, e o resultado dessa soma foi dividido pela perda total deágua no período, para cada tratamento.

As perdas de $\mathrm{N}$ e CO foram obtidas pela multi plicação da concentração com o volume perdido, em cada evento. A soma das perdas em cada evento resultou no val or de perda acumulada no período, ou seja, maio/2006 a maio/2008. A percentagem de perdas de $\mathrm{NO}_{3}-\mathrm{e} \mathrm{NH}_{4}{ }^{+}$foi cal culada considerando a perda acumulada no período de ambas as formas de N. Em razão de o vol ume col etado não ser suficiente para todas as análises, houve quantidades diferentes de amostras analisadas: 440 para o $\mathrm{NH}_{4}{ }^{+}, 318$ para o $\mathrm{NO}_{3}{ }^{-}$e 299 para a determinação de COT.

Foi realizada a análise de variância consi derando a regressão para avaliar o efeito das doses de dejeto líqui do bovino nas concentrações médias ponderadas e nas perdas acumuladas no período (Gomes, 1987), utilizando-seosoftwareSTATGRAPHICS ${ }^{\circledR}$ Centurion VX, versão 15.1.02.

\section{RESULTADOS E DISCUSSÃO}

\section{Concentração média ponderada e perda de carbono orgâni co total}

A aplicação de dejeto líquido bovino não alterou significativamente o comportamento do carbono orgânico total (COT) no escoamento superficial. No entanto, em estudo similar a este, porém em solo de textura franco-argilo-arenosa, Silveira et al. (2011) observaram diferenças significativas; a aplicação de $120 \mathrm{~m}^{3}$ ha-1 $^{-1}$ ano $^{-1}$ reduziu em 20 e $89 \%$ a concentração média ponderada ea perda deCOT, respectivamente, em relação ao tratamento sem aplicação. Essa diferença decomportamento no $\mathrm{CO}$ entreos dois tipos de sol o deve-se possivel mente ao efeito positivo nos atributos físicos do sol o com a aplicação de dejeto no solo franco-argilo-arenoso (Mellek et al., 2010), diminuindo significativamente as perdas de solo e água e, consequentemente, de CO.

As concentrações médias ponderadas de COT (Figura 1a), o qual é constituído por CO solúvel epor CO particulado, apresentaram comportamento semel hante ao das perdas de solo, as quais também não foram diferentes estatisticamente (Timofiecsyk, 2009), indicando maior contribuição do C associado ao sedimento (fração particulada) em relação à fração solúvel.

A quantidadeperdida deC dependeda concentração de C no escoamento etambém da quanti dade perdida de água; de acordo com a figura $1 b$, as perdas acumuladas de COT mostraram comportamento semel hanteao das perdas deágua (Timofiecsyk, 2009), indicando maior efeito da quantidade deágua perdida do que da concentração de C. N o entanto, as perdas de água, diferentemente das perdas de $C$, diferiram estatisticamente entre os tratamentos, tendo sido menores na dose de $120 \mathrm{~m}^{3} \mathrm{ha}^{-1} \mathrm{ano}^{-1}$ de dejeto líquido bovino (Timofiecsyk, 2009). Para o tratamento sem aplicação do dejeto, o C perdido é proveniente da matéria orgânica do sol o(MOS); apesar da nãoadição de dejeto, estetratamento apresentou a maior perda acumulada de C (Figura 1b). Isso ocorreu devido à maior perda deágua nestetratamento (Timofiecsyk, 2009). Esses resultados evidenciam queem áreas com PD, mesmo sem aplicação de dejeto, práticas conservacionistas deveriam ser utilizadas a fim de conter o escoamento na própria lavoura, evitando a entrada de contaminantes associados ao escoamento em cursos deágua (Bertol et al., 2005; Shigaki et al., 2006).

A presença de CO na água gera problemas, uma vez que ele será mineralizado, reduzindo o $\mathrm{O}_{2}$ dissolvido. Além disso, a mineral ização da MOS está associada coma disponibilização de N eP (E ghball et al., 2002). Para C solúvel, a preocupação refere-seà formação de compostos cancerígenos no processo de cloração, em sistemas de tratamento de água (Kay et al., 2009).

\section{Concentração média ponderada e perdas de $\mathrm{N}-\mathrm{NO}_{3}{ }^{-}$e N-NH${ }_{4}^{+}$}

As concentrações médias ponderadas de $\mathrm{N}$ tanto na forma de $\mathrm{NO}_{3}{ }^{-}$como de $\mathrm{NH}_{4}{ }^{+}$(Figuras $1 \mathrm{c}$,e) foram mai ores com a aplicação de dejeto, atingindo val ores máximos na maior dose (180 $\left.\mathrm{m}^{3} \mathrm{ha}^{-1} \mathrm{ano}^{-1}\right)$, o que ilustra o potencial poluidor de resíduos orgânicos, principal mente os aplicados na superfície do solo, em áreas agrícolas sob PD (Smith et al., 2001; Allen \& Mallarino, 2008; Silveira et al., 2011). As concentrações médias ponderadas de $\mathrm{NH}_{4}{ }^{+}$em todos os tratamentos, inclusive o sem aplicação de dejeto, ul trapassaram o limite permitido pela legislação (CONAMA, 2005), a qual estabelece olimite de $\mathrm{N}-\mathrm{NH}_{4}{ }^{+}$de $3,7 \mathrm{mg} \mathrm{L}^{-1}$ para um $\mathrm{pH}$ em água de aproximadamente 7,5. Considerando olimite permitido de $\mathrm{NO}_{3}{ }^{-}\left(10 \mathrm{mg} \mathrm{L}^{-1} \mathrm{de}\right.$ $\mathrm{N}-\mathrm{NO}_{3}{ }^{-}$) (Brasil, 2004; Conama, 2005), observa-seque até a dose de $120 \mathrm{~m}^{3} \mathrm{ha}^{-1} \mathrm{ano}^{-1}$ a concentração média ponderada de $\mathrm{NO}_{3}{ }^{-}$, na água do escoamentosuperficial, está abaixo do limitemáximo permitido por legislação; no entanto, a dose máxima de dejeto líquido bovino ultrapassou em 36,4 \% estelimite. Apesar dos baixos 

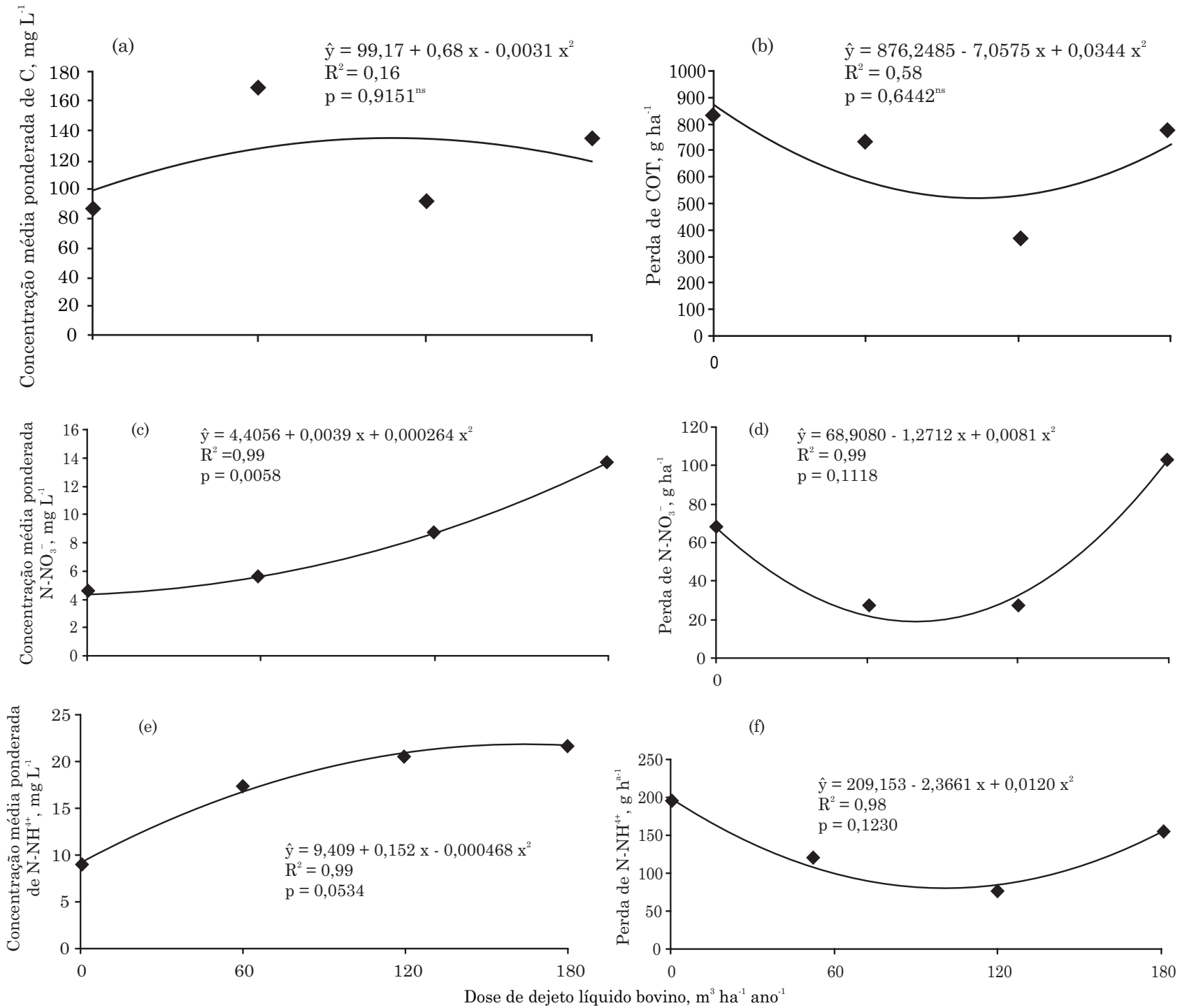

(f)

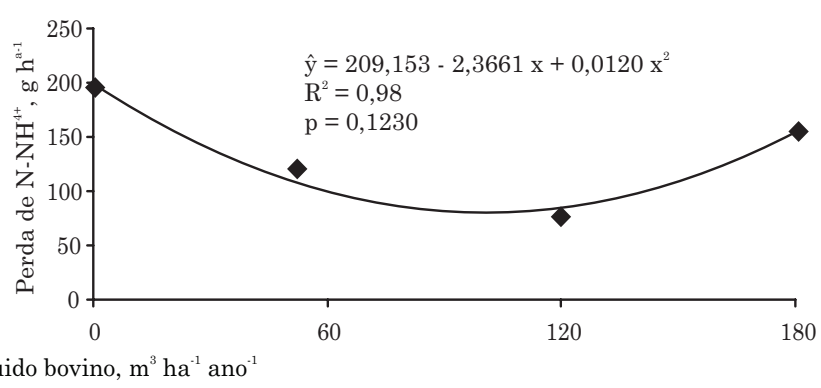

Figura 1. Concentração média ponderada de carbono orgâni co total (a), de N-nitrato (c), de N-amônio (e) e perda de carbono total (b), de N-Nitrato (d), de N-amônico (f) em escoamento superficial com aplicação de dejeto líquido bovino em Latossolo Bruno muito argi loso sob plantio direto echuva natural no período de maio de 2006 a maio de 2008.

val ores demédia ponderada, éinteressanteressaltar que a concentração de $\mathrm{NO}_{3}^{-}$, em várias amostras, ultrapassou olimitepermitido pela legislação, mesmo no tratamento sem dejeto, mostrando o potencial poluidor dessesistema de produçãoe ea necessi dade de práticas conservacionistas a fim de controlar o escoamento eevitar a entrada desteem corpos deágua.

Noentanto, apesar doaumento das concentrações, as perdas acumuladas de $\mathrm{NO}_{3}{ }^{-}$(Figura $1 d$ ) e $\mathrm{NH}_{4}{ }^{+}$ (Figura 1f) reduziram com a aplicação de dejeto atéa dose de $120 \mathrm{~m}^{3} \mathrm{ha}^{-1} \mathrm{ano}^{-1}$, estando positivamente relacionadas com as perdas de água (Timofiecsyk, 2009); isso está de acordo com os dados de Ceretta et al. (2005) e Mori et al. (2009), indicando ser essa a melhor dose de aplicação, pois evita problemas ambientais eao mesmotempo possi bilita a reciclagem de resíduos orgânicos em áreas agrícolas.

A aplicação de dejeto em longo prazo promove a melhoria da estrutura física do solo e, em consequência, aumento da infiltração e redução de perdas de sol o eágua (Mellek et al., 2010); contudo, em curto prazo, a aplicação de dejeto líquido pode propiciar selamentosuperficial eimpedir a infiltração deágua nosolo (Oliveira et al., 2000; M ori et al., 2009), aumentando consequentementeas perdas deágua e nutrientes (Smith et al., 2001).

Comparando as quantidades perdidas de $\mathrm{NH}_{4}{ }^{+} \mathrm{e}$ de $\mathrm{NO}_{3}$ - (Figura 2), observam-se maiores perdas de $\mathrm{NH}_{4}{ }^{+}$do que de $\mathrm{NO}_{3}{ }^{-}$- comportamento também observado por Silveira et al. (2011). Isso ocorreu, 


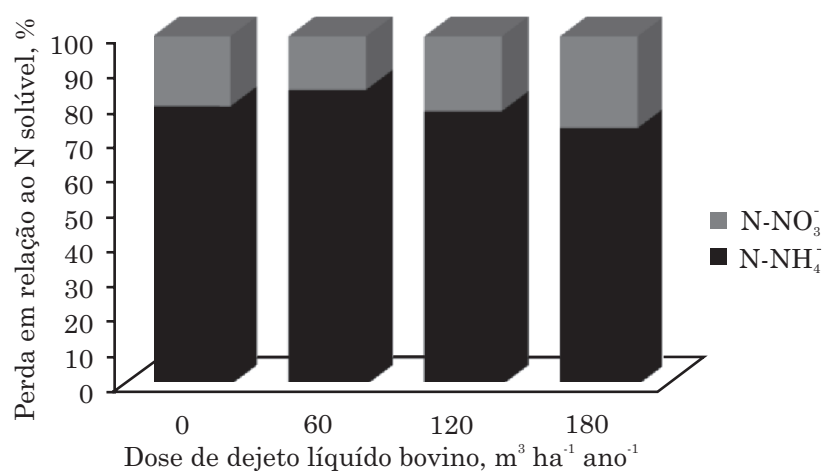

Figura 2. Relação das perdas de $\mathrm{N}$-nitrato e $\mathrm{N}$-amônio em escoamento superficial com aplicação de dejeto líquido bovino em Latossolo Bruno muito argiloso sob plantio direto e chuva natural no período de maio de 2006 a maio de 2008.

provavel mente, devido à maior aplicação de $\mathrm{NH}_{4}{ }^{+}$do que de $\mathrm{NO}_{3}{ }^{-}$via dejeto etambém pela dinâmica desses elementos no solo. Estima-se que nos dejetos armazenados anaerobicamente - e portanto sem possibilidade de nitrificação - cerca de $70 \%$ do N inorgânico esteja na forma amoniacal, ou seja, $\mathrm{N}$ $\mathrm{NH}_{4}{ }^{+}+\mathrm{N}-\mathrm{NH}_{3}{ }^{-}$(Scherer et al., 1996). Além disso, o $\mathrm{NH}_{4}{ }^{+}$tende a ser adsorvido nas partículas e, assim, perde-semais por processos erosivos, enquanto $\mathrm{NH}_{3}$, em razão da sua baixa reatividade eal ta mobilidade, é preferencial mente perdido por lixiviação (Sposito, 1989). A maior perda de $\mathrm{NH}_{3}{ }^{-}$foi na dose de $180 \mathrm{~m}^{3}$ ha-1 $\mathrm{ano}^{-1}$, evidenciando que ele, além de ser perdido por lixiviação, também podeser perdido pela erosão via escoamento superficial. Esse fato pode ser explicado pela alta concentração de compostos gordurosos nos dejetos (Dinel et al., 1998), o que dificulta a infiltração deágua nosolo, propiciandomaior volume do escoamento superficial potencialmenterico em nutrientes. Notratamento sem aplicação de dejeto observam-setambém el evadas perdas, o queevidencia o potencial detransporte denutrientes em sistemas de plantio direto devido ao acúmulo destes na superfície dosol o (Bertol et al., 2007b).

\section{CONCLUSÕES}

1. A aplicação do dejetolíquido bovino diminuiu as quantidades perdidas deamônio enitrato atéa dose de $120 \mathrm{~m}^{3} \mathrm{ha}^{-1}$ ano-1, o que indica ser esta a dose máxima recomendada para sol o muito argiloso, dedive moderadamente ondulado, baixas precipitações pluviais e com no mínimo uma semana de intervalo entre a aplicação do dej eto e a ocorrência de chuvas. O potencial poluidor podeaumentar com sucessivas aplicações, havendo a necessidade de estudos superiores a dois anos de aplicação.

2. As concentrações de amônio estiveram muito acima do máximo permitido pela legisl ação em todos os tratamentos, indicando a necessi dade de técnicas conservacionistas que aumentem a infiltração do escoamento superficial na área agrícola, mesmo em plantio direto, evitando assim a entrada de contaminantes associados ao escoamento no sistema aquático.

\section{LITERATURA CITADA}

ALLEN, B.L. \& MALLARINO, A.R. Effect of liquid swine manure rate, incorporation, and timing of rainfall on phosphorus loss with surface runoff. J. Environ. Qual., 37:125-137, 2008.

AMERICAN PUBLIC HEALTH ASSOCIATION - APHA. Standard methods for the examination of water and wastewater. 19.ed. Washington D.C., American Public Health Association, 1995.

BERTOL, O.J .; PAULETTI, V. \& DIECKOW, J . A transferência de tecnologia em manejo e conservação do solo e da água. Bol. Inf. SBCS, 37:26-31, 2012.

BERTOL, O.J .; RIZZI, N.E.; FAVARETTO, N. \& LAVORANTI, O.J. Perdas de nitrogênio via superfície e subsuperfície em sistema de semeadura direta. R. Flor., 35:429-443, 2005.

BERTOL, O.J .; RIZZI, N.E.; BERTOL, I.\& ROLOFF, G. Perdas de solo e água e qualidade do escoamento superficial associadas à erosão entre sulcos em área cultivada sob semeadura direta e submetida às adubações mineral e orgânica. R. Bras. Ci. Solo, 31:781-792, 2007 a.

BERTOL, I.; ENGEL, F.L.; MAFRA, A.L.; BERTOL, O.J . \& RITTER, S.R. Phosphorus, potassium and organic carbon concentrations in runoff water and sediments under different soil tillage systems during soybean growth. Soil Tillage Res., 94:142-150, 2007b.

BRASIL. Ministério da Saúde. Secretaria de Vigilância em Saúde, Coordenação-Geral de Vigilância em Saúde Ambiental. Portaria MS no 518/2004. Brasília, 2004.

CONSELHO NACIONAL DO MEIO AMBIENTE - CONAMA. Resolução CONAMA no 357. Diário Oficial da União de 18 de março de 2005. Brasília, 2005.

BOYD, C. \& TUCKER, C. Water quality and pond soil analyses for aquaculture. Alabama, Auburn University, 1992. 183p.

CERETTA, C.A.; BASSO, C.J .; VIEIRA, F.C.B.; BARCELLOS, L.A.R.; HERBES, M.G.; MOREIRA, I.C.L. \& BERWANGER, A.L. Dejeto líquido de suínos: I - Perdas de nitrogênio e fósforo na solução escoada na superfície do solo, sob plantio direto. Ci. Rural, 35:1296-1304, 2005.

COGO, N.P.; LEVIEN, R. \& SCHWARZ, R.A. Perda de solo e água por erosão hídrica influenciadas por métodos de preparo, classes de declive e níveis de fertilidade do solo. R. Bras. Ci. Solo, 27:743-753, 2003.

CORRELL, D.L. The role of phosphorus in the eutrophication of receiving waters: A review. J. Environ. Qual., 27:261266, 1998. 
DANIEL, T.C.; SHARPLEY, A.N. \& LEMUNYON, J.L. Agricultural phosphorus and Eutrophication: A Symposium Overview. J . Environ. Qual., 27:251-257, 1998.

DERPSCH, R.; ROTH, C.H.; SIDIRAS, N. \& KOPKE, U. Controle da erosão no Paraná, Brasil: Sistemas de cobertura do solo, plantio direto e preparo conservacionista do solo. Eschborn, Deutsche Gesellschaft fur Technische Zusammenarbeit, 1991. 272p.

DINEL, H.; SCHNITZER, M. \& SCHULTEN, H.R. Chemical and spectroscopic characterization of colloidal fractions separated from liquid hog manures. Soil Sci., 163:665673, 1998.

DINNES, D.L.; KARLEN, D.L.; J AYNES, D.B.; KASPAR, T.C.; HATFIELD, J .L.; COLVIN, T.S. \& CAMBARDELLA, C.A. Nitrogen management strategies to reduce nitrate leaching in tile-drained midwestern soils. Agron. J ., 94:153-171, 2002.

EGHBALL，B.; WIENHOLD，B.J .; GILLEY, J.E. \& EIGENGERG, R.A. Mineralization of manure nutrients. J. Soil Water Conserv., 57:470-473, 2002.

EMPRESA BRASILEIRA DE PESQUISA AGROPECUÁRIA EMBRAPA/F undação ABC. Mapa do levantamento semidetal hado desol os: Muniápio de Castro. Castro, PR. 2001.

GOMES, F.P. Curso de estatística experimental. Piracicaba, USP-ESALQ, 1987. 467p.

HOODA, P.S.; EDWARDS, A.C.; ANDERSON, H.A. \& MILLER, A. A review of water quality concerns in livestock farming areas. Sci. Total Environ., 250:143-147, 2000.

INSTITUTO AGRONÔMICO DO PARANÁ - IAPAR. Monitoramento agroclimático. Disponível em: «tttp:// www.iapar.br/arquivos/l mage/monitoramento/ Medias_Historicas/Ponta_Grossa.htm>. Acesso em julho de 2012.

KAY, P.; EDWARDS, A.C. \& FOULGER, M. A review of the efficacy of contemporary agricultural stewardship measures for ameliorating water pollution problems of key concern to the UK water industry. Agric. Syst., 99:6775, 2009.

MARTINS, A.P.L. \& REISSMANN, C.B. Material vegetal e as rotinas laboratoriais nos procedimentos químicoanalíticos. Sci. Agríc., 8:1-17, 2007.

MELLEK, J .E.; DIECKOW, J .; SILVA, V.L.; FAVARETTO, N.; PAULETTI, V.; VEZZANI, F.M. \& SOUZA, J.L. Dairy liquid manure and no-tillage: Physical and hydraulic properties and carbon stocks in a Cambisol of Southern Brazil. Soil Tillage Res., 110:69-76, 2010.

MORI, H.F.; FAVARETTO, N.; PAULETTI, V.; DIECKOW, J . \& SANTOS, W.L. Perdas de água, solo e fósforo com aplicação de dejeto líquido bovino em $L$ atossolo sob plantio direto e chuva simulada. R. Bras. Ci. Solo, 33:189-198, 2009.

OLIVEIRA, R.A.; CAMPELO, P.L.G.; MATOS, A.T.; MARTINEZ, M.A. \& CECON, P.R. Influência da aplicação de águas residuárias de suinocultura na capacidade de infiltração de um solo Podzólico VermelhoAmarelo. R. Bras. Eng. Agríc. Amb., 4:263-267, 2000.
POTE, D.H.; DANIEL, T.C.; NICHOLS, D.J .; SHARPLEY, A.N.; MOORE, P.A.; MILLER, D.M. \& EDWARDS, D.R. Relationship between phosphorus levels in three Ultisols and phosphorus concentrations in runoff. J. Environ. Qual., 28:170-175, 1999.

RESCK, D.V.S.; FIGUEIREDO, M.S.; FERNANDES, B.; RESENDE, M. \& SILVA, T.C.A. Intensidade de perdas de nutrientes em um Podzólico Vermelho-Amarelo, utilizando-se simulador de chuva. R. Bras. Ci. Solo, 4:188192, 1980.

SCHERER, E.E.; AITA, C. \& BALDISSERA, I.T. Avaliação da qualidade do esterco líquido de suínos na região oeste catarinense para fins de utilização como fertilizante. Florianópolis, EPAGRI, 1996. (Boletim Técnico, 79)

SHARPLEY, A.N.; CHAPRA, S.C.; WEDEPOHL, R.; SIMS, J .T.; DANIEL, T.C.\& REDDY, K.R. Managing agricultural phosphorus for protection of surface waters: Issues and options. J . Environ. Qual., 23:437-451, 1994.

SHARPLEY, A.N. I dentifying sites vulnerable to phosphorus loss in agricultural runoff. J . Environ. Qual., 24:947-951, 1995.

SHIGAKI, F.; SHARPLEY, A.N. \& PROCHNOW, L.I. Animalbased agriculture, phosphorus management and water quality in Brazil: options for the future. Sci. Agríc., 63:194209, 2006.

SILVA, D.D.; PRUSKI, F.F.; SCHAEFER, C.E.G.R.; AMORIM, R.S.S. \& PAIVA, K.W.N. Efeito da cobertura nas perdas de solo em um Argissolo Vermel ho-Amarelo utilizando simulador de chuva. Eng. Agríc., 25:409-419, 2005.

SILVEIRA, F.M.; FAVARETTO, N.; DIECKOW, J .; PAULETTI, V.; VEZZANI, F.M. \& SILVA, E.D. Dejeto líquido bovino em plantio direto: Perda de carbono e nitrogênio por escoamento superficial. R. Bras. Ci. Solo, 35:1759-1767, 2011.

SMITH, S.J .; SCHEPERS, J .S. \& PORTER, L.K. Assessing and managing agricultural nitrogen losses to the environment. In: STEWART, B.A., ed. Advances in soil science. Chelsea, Lewis Publishers, 1990. v.14. p.1-43.

SMITH, V.H.; TILMAN, G.D. \& NEKOLA, J.C. Eutrofication: impacts of excess nutrient inputs on freschwater, marine, and terrestrial ecosystems. Environ. Poll., 100:179-196, 1999.

SMITH, K.A.; J ACKSON, D.R. \& PEPPER, T.J . Nutrient losses by surface run-off following the application of organic manures to arable land. I. Nitrogen. Environ. Poll., 112:4151, 2001.

SPOSITO, G. The chemistry of soils. New York, Oxford University Press, 1989. 277p.

TIMOFIECSYK, A. Perdas de água, solo e nutrientes com aplicação de dejeto líquido bovino sob plantio direto e chuva natural em Latossolo Bruno. Curitiba, Universidade Federal do Paraná, 2009. (Tese de Mestrado) 